\title{
INCLUSION COMPOUNDS OF $\alpha$-CYCLODEXTRIN WITH ALKYLTHIOLS
}

\author{
PAUL JARA ${ }^{1 *}$, LORENA BARRIENTOS ${ }^{1}$, BARBARA HERRERA ${ }^{1}$ and ISABEL SOBRADOS ${ }^{2}$ \\ ${ }^{1}$ Departamento de Química, Facultad de Ciencias, Universidad de Chile, Santiago, Chile, \\ ${ }_{2}^{2}$ Instituto de Ciencia de Materiales de Madrid, Consejo Superior de Investigaciones Cientificas (CSIC), Cantoblanco 28049 Madrid, Spain \\ (Dedicated in memorial of our colleague and friend Prof. Dr. Jaime Retuert) \\ (Received: 20 December 2007 - Accepted: 12 March 2008)
}

\begin{abstract}
We report the formation of $\alpha$-cyclodextrin $(\alpha \mathrm{CD})$ inclusion compounds with octanethiol (OT), decanethiol (DT) and dodecanethiol (DDT). Elemental Microanalysis, ${ }^{1} \mathrm{H}-\mathrm{NMR}$ solution, Scanning Electronic Microscopy (SEM) and Powder X-ray Diffraction analysis (PXRD) confirm the inclusion process of the alkylthiols in to the $\alpha$-cyclodextrin molecules. The basic host structure of the products is similar to that of typical channel type structure cyclodextrin inclusion compounds. The guest presents extended linear (zig-zag) conformation. The presence of the $-\mathrm{SH}$ groups located in the (001) plane of the $\alpha$-cyclodextrinsalkylthiol crystal, is evidenced by Energy dispersive X-ray (EDX) analysis. ${ }^{13} \mathrm{C}$ CP-MAS NMR spectra of new $\alpha$-cyclodextrin host-guest inclusion compounds are described.
\end{abstract}

Keywords: Inclusion compound, Cyclodextrin, Thiol

\section{INTRODUCTION}

$\alpha, \beta$ or $\gamma$-cyclodextrins are cyclic oligosaccharides consisting in 6,7 and 8 glucopyranose units, respectively. These compounds have relatively rigid doughnut-shaped structures, and constitute very useful monomolecular hosts in supramolecular chemistry ${ }^{1,2}$. Molecules of adequate size, shape and suitable functionality can be held within the cavity of a particular cyclodextrin The inclusion complexation occurs when the electrostatic, van der Waals, hydrophobic interactions and hydrogen bonding ${ }^{3}$ are cooperative. The cyclodextrins and their inclusion compounds can be crystallized from water and studied by X-ray crystallography as empty molecules or as host-guest complexes. "Channel" or "cages" structures are formed depending of the size and ionic or molecular character of the substrate, in which the cyclodextrin molecules are stacked $\mathrm{d}^{2,4-5}$.

The cyclodextrin inclusion compounds, particularly the ones leading to supramolecular self-assemblies continue to be a fascinating topic in modern organic chemistry as they serve as models for understanding molecular recognition and as precursors for designing novel nanomaterials ${ }^{6}$ for electronics and biological applications ${ }^{7}$. On the other hand, renewed interest in metal clusters and colloidal particles has given rise to an expanding area of interdisciplinary research. For example, gold colloidal particles can be modified with cyclodextrin like molecular receptor ${ }^{8,9}$

Previously, we reported the synthesis and structural properties of cyclodextrin dialkyl- ${ }^{8}$, alkyl- ${ }^{-9}$ and bicyclic-amine ${ }^{10}$ inclusion compounds showing channel structures with a hexagonal lattice. ${ }^{13} \mathrm{C}-\mathrm{MAS}$ NMR spectra show different resonance signals for the homologous carbon atom of both dialkylamine branches that evidences the non-symmetric location of the amine in the cyclodextrin matrix channel.

In this work we report the preparation of $\alpha$ - cyclodextrins solid inclusion compounds with octanethiol, decanethiol and dodecanethiol.

\section{EXPERIMENTAL}

Commercially available reagents were used as received.The inclusion compounds were obtained from alkylthiols (OT, DT and DDT) and saturated solutions of $\alpha$-cyclodextrin in water at room temperature. The thiol: cyclodextrin molar ratios used in the experiments were always somewhat greater than the stoichiometric ratio found for the products. Microcrystals are separated immediately, washed with acetone and dried under vacuum at room temperature. The molar ratio between $\mathrm{CD}$ and thiols were determined by ${ }^{1} \mathrm{H}-\mathrm{NMR}$ of dimethyl- $\mathrm{d}_{6}$ sulfoxide solutions and elemental analysis (Perkin Elmer $240 \mathrm{C}$ microanalyzer). The microcrystals were analyzed by powder Xray diffraction (PXRD). Powder X-ray diffraction pattern of $\alpha$-CD showed that obtained phase corresponded to channel-type structure with the main reflections at $2 \theta=19.90^{\circ}(\mathrm{d}=4.46 \AA)^{9}$. The following stoichiometries were determined: $\alpha \mathrm{CD} / \mathrm{OT}, 2 \alpha \mathrm{CD} / \mathrm{DT}$ and $2 \alpha \mathrm{CD} / \mathrm{DDT}$.

Scanning Electron Microscopy (SEM) was realized in a Phillips EM100 Microscopy. Solution ${ }^{1} \mathrm{H}$ high resolution NMR spectra were recorded on a Bruker AMX-300. Powder X-ray diffractograms were recorded in the range $2^{\circ}$ $<2 \theta<50^{\circ}$ on a Siemens D-5000 diffractometer using $\mathrm{Cu}-\mathrm{K}_{\alpha}$ radiation $(40 \mathrm{KV}$,
$30 \mathrm{~mA})$ and a graphite monochromator $(\lambda=1.540598 \AA)$. Samples of $\alpha \mathrm{CD} / \mathrm{OT}$, $2 \alpha \mathrm{CD} / \mathrm{DT}$ and $2 \alpha \mathrm{CD} / \mathrm{DDT}$ inclusion compounds were ground to a fine powder in order to reduce the likelihood of the crystallites exhibiting a preferred orientation. For the two products the diffracrtograms indicated absence of any other crystalline phases than those of the reported inclusion compounds.

\section{RESULTS AND DISCUSSION}

Analytical as well as further characterization of the products clearly show that thiols $\mathrm{CH}_{3}\left(\mathrm{CH}_{2}\right)_{\mathrm{n}} \mathrm{SH}$ with $\mathrm{n}=7,9,11$ can be accommodated by $\alpha$ cyclodextrin matrix to form stable inclusion compounds with channel structures at room temperature which are similar to those obtained from the inclusion of the other guest ${ }^{8-10}$. In this inclusion compounds the thiol guest, occupy one cyclodextrin units for OT and two cyclodextrin units for DT and DDT. The three guests present probably extended linear (zig-zag) conformation. The space periodicity along the prism axis, calculated from spacing of cyclodextrin matrix crystallographic layer line, is comparable to the predicted length of the guests $(10.26 \AA$ for OT, $12.80 \AA$ for DT and $15.28 \AA$ for DDT, in extended linear conformation). The cyclodextrin molecules take the shape of a cone with $\mathrm{C} 2$ and $\mathrm{C} 3$ hydroxyl groups located around the larger opening and the more reactive C6 hidroxyl aligned around the smaller opening ${ }^{1-3}$. The arrangement of C6 hydroxyl opposite to the hydrogen bonded $\mathrm{C} 2$ and $\mathrm{C} 3$ hidroxyl forces the oxygen bonds into close proximity within the cavity, leading to an electron rich, hydrophobic interior. In cyclodextrin inclusion compounds the matrix channel is formed by these cones unities which interact through van der Waals forces and are ordered to encounter the similar end of neighbouring units (larger opening-larger opening, smaller opening-smaller opening). In the inclusion of alkylthiol guest, the -SH group of the guest could be located at the extreme boundary of a cyclodextrin unit, in the rich electron space density with the alkyl chain located in the apolar and poor electron density zone of the cyclodextrins cavities. Fig. 1 shows a schematic representation of the inclusion of DT in cyclodextrin. Crystal inspection by Scanning Electron Microscopy (SEM) and Energy dispersive X-ray (EDX) analysis microcrystal of $\alpha \mathrm{CD}$ inclusion compound confirms the preferential location of - $\mathrm{SH}$ groups from the guest molecules found within the CD protrude in the $\{001\}$ crystal plane (Fig. 2). These -SH groups form a two-dimensional hexagonal lattice on the structures of the $\alpha$-CD-alkylthiol inclusion compounds. This morphology is consistent with the predicted interaction between hidroxyl and the - $\mathrm{SH}$ group, located in the $\{001\}$ plane of the $\alpha$-cyclodextrins-alkylthiol inclusion compound microcrystal. All peaks in the diffractograms, can be indexed on the basis of a hexagonal lattice with parameter values close to $\mathrm{a}=\mathrm{b} \approx 27 \AA$, $\mathrm{c} \approx 16 \AA \AA, \alpha=\beta$ $=90^{\circ}$ and $\gamma=120^{\circ}$ for $\alpha$-cyclodextrin ${ }^{8}$. A typical indexed diffractograms for the inclusion compounds is shown in Fig. 3. 




Figure 1. Approximated representation of $\alpha \mathrm{CD} / \mathrm{DT}$ inclusion compound.

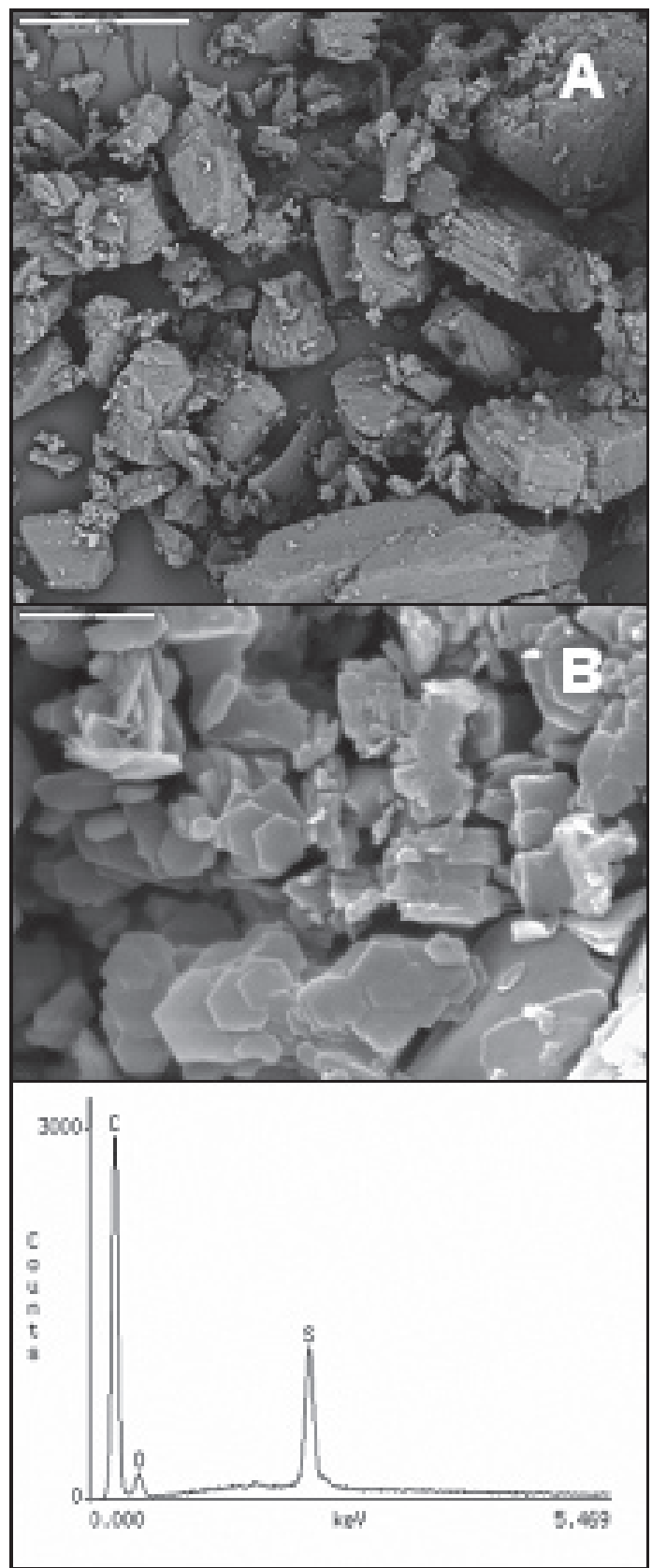

Figure 2. SEM image reveals the change in morphology between a) pure $\alpha \mathrm{CD}$ and $\mathrm{b}$ ) the complex formed with EDX analysis.

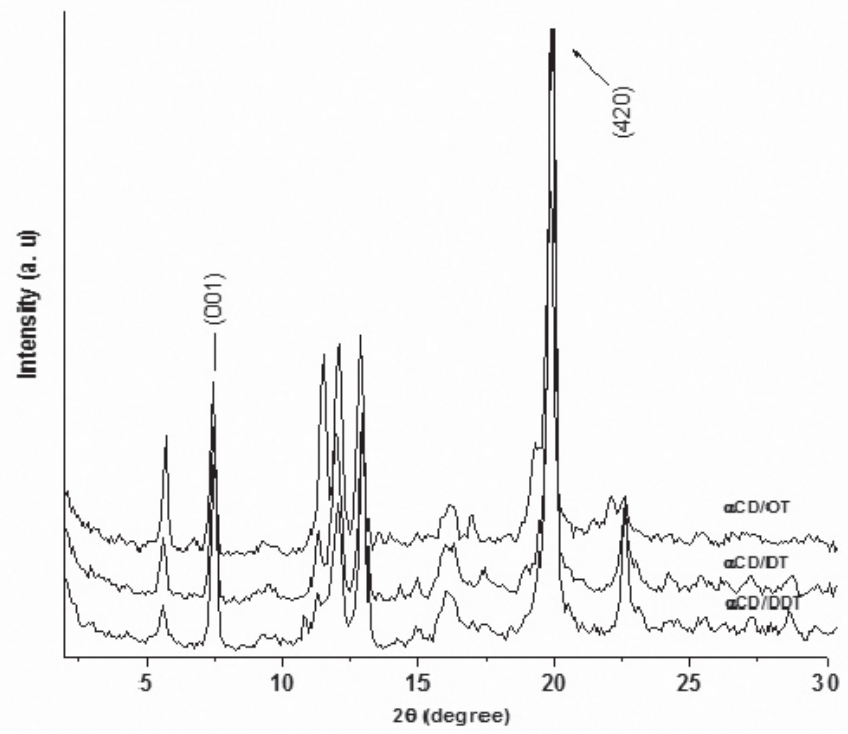

Figure 3. PXRD patterns for $\alpha \mathrm{CD} / \mathrm{OT}, 2 \alpha \mathrm{CD} / \mathrm{DT}$ and $2 \alpha \mathrm{CD} / \mathrm{DDT}$ inclusion

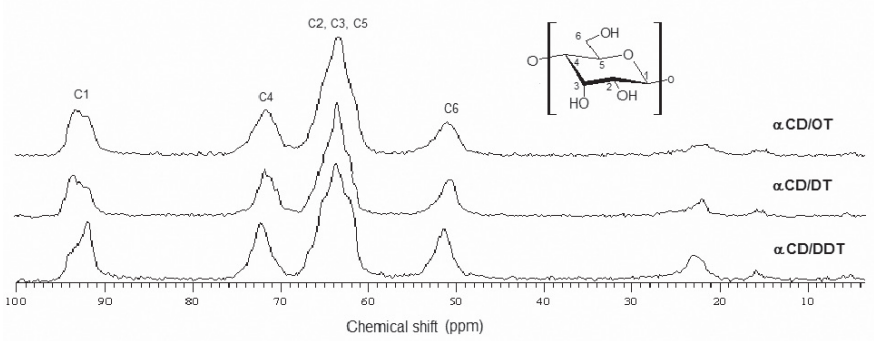

Figure 4. ${ }^{13} \mathrm{C}$ CP MAS RMN spectra for samples of $\alpha \mathrm{CD}$ inclusion compounds.

${ }^{13} \mathrm{C}$ CP-MAS NMR spectra of acyclodextrin inclusion compounds are recorder. Chemical shifts of the confined guest molecules in inclusion compounds reveal different resonance signals for the homologous carbon atoms. For the guests a disorder in cyclodextrin inclusion compounds is observed probably in the extended linear (zig-zag) conformation.

From the results discussed above, it can be concluded that cyclodextrin form stable inclusion compound with OT, DT and DDT and generate a well defined surface functionality of $\mathrm{CD}$ inclusion compounds, where the surface functional groups $-\mathrm{SH}$ can be adjusted by the guest molecules for potential applications in supramolecular and nano chemistry. ${ }^{11}$

\section{ACKNOWLEDGEMENTS}

Financial support by FONDECYT (Grants 1040581) is gratefully acknowledged.

We also thank MECESUP-UMCE for a fellowship to L.B.P.

\section{REFERENCES}

1. J. Szejtli, Chem. Rev., 98, 1743, (1998); G. Wenz, B. Han, and A. Muller, Chem Rew, 106, 782, (2006).

2. K. Harata, Chem. Rev., 98, 1803, (1998).

3. L. Liu, O. Gou, J .Incl. Phenom., 42, 1, (2002).

4. W. Saenger, Angew. Chem. Int. Eng., 19, 344, (1980).

5. K. Takemoto, Sonoda, N. in Inclusion Compounds, J.L. Atwood, J.E.D. Davies and D.D. MacNicol eds. Academic Press, New York, (1984).

6. R. Wassel, G. Credo, R. Fuierer, D. Feldheim, C. Gorman, J. Am. Chem. Soc., 126(1), 295, (2004). 
7. J. Liu, X. Ni, Z. Zhou, K.W. Leong, J. Am. Chem. Soc., 125(7), 1788, (2003).

8 P. Jara, M. Justiniani, N. Yutronic, I. Sobrados, J .Incl. Phenom., 32, 1, (1998)

9. P. Jara, X. Cañete, N. Yutronic, J.Chil. Chem. Soc., 49, 241, (2004).
10. N. Yutronic, X. Cañete, P. Jara, W. Lavayen, Mol. Cryst. Liq. Cryst., 417, 193, (2004)

11. L. Barrientos, N. Yutronic, F. del Monte, M.C. Gutierrez and P. Jara. New J. Chem., 31, 1400, (2007). 